\title{
Radiosensitization of Glioblastoma Cells by a Novel DNA Methyltransferase-inhibiting Phthalimido-Alkanamide Derivative
}

\author{
CHAN WOO WEE ${ }^{1}$, JIN HO KIM ${ }^{1,2,3}$, HAK JAE KIM ${ }^{1,2,3}$, HYUN-CHEOL KANG ${ }^{1}$, \\ SOO YOUN SUH ${ }^{2}$, BEOM SOO SHIN ${ }^{4}$, EUNSOOK MA ${ }^{5}$ and IL HAN KIM ${ }^{1,2,3}$ \\ ${ }^{1}$ Department of Radiation Oncology, Seoul National University College of Medicine, Seoul, Republic of Korea; \\ ${ }^{2}$ Cancer Research Institute, Seoul National University College of Medicine, Seoul, Republic of Korea; \\ ${ }^{3}$ Institute of Radiation Medicine, Seoul National University College of Medicine, Seoul, Republic of Korea; \\ ${ }^{4}$ School of Pharmacy, Sungkyunkwan University, Seoul, Republic of Korea; \\ ${ }^{5}$ College of Pharmacy, Catholic University of Daegu, Gyeongsan, Republic of Korea
}

\begin{abstract}
Background/Aim: Strategies to enhance the therapeutic ratio of radiotherapy in glioblastoma are warranted. Our aim was to report a novel DNA methyltransferase inhibitor as a potential radiosensitizing agent in glioblastoma. Materials and Methods: Four glioblastoma cell lines and one normal astrocyte cell line were incubated with a newly-synthetized phthalimidoalkanamide derivative, MA17, and its radiosensitizing effects were assessed. We performed a tumor growth delay assay in two glioblastoma lines: U87MG and U138MG. We evaluated DNA methyltransferase (DNMT) inhibition, apoptosis, autophagy, DNA damage repair, and FANCA expression. Results: MA17 radiosensitized all glioblastoma cells (all $p<0.05)$, but it did not affect normal astrocytes ( $p=0.193)$. MA17 significantly prolonged the mean tumor doubling time in vivo, in cells treated in addition with radiotherapy, compared to radiotherapy alone $(p<0.05)$. DNMT activity was down-regulated, and apoptosis and autophagy were induced by MA17. Double-stranded DNA break foci were observed for prolonged periods in cells treated with MA17. FANCA expression was also inhibited. Conclusion: A novel
\end{abstract}

Correspondence to: Il Han Kim, Department of Radiation Oncology, Cancer Research Institute, Institute of Radiation Medicine, Seoul National University College of Medicine, 101 Daehak-ro Jongno-gu, Seoul 03080, Republic of Korea. Tel: +82 220722528, Fax: +82 27653317, e-mail: ihkim@snu.ac.kr and EunSook Ma, College of Pharmacy, Daegu Catholic University, Hayang-Ro 13-13, Hayang-Eup, Gyeongsan-si, Gyeongbuk, 38430, Republic of Korea. Tel: +82 538503621, Fax: +82 538503602, masook@cu.ac.kr

Key words: DNA mehyltransferase, radiotherapy, radiosensitizer, glioblastoma, phthalimido-alkanamide. phthalimido-alkanamide derivative demonstrated significant radiosensitization in glioblastoma cells in vitro and in vivo. Further investigation is needed to translate these results to the clinic.

Glioblastoma (GBM), the most common brain cancer in adults, with a devastating survival of 15-20 months for patients despite standard chemoradiation with temozolomide $(1,2)$. In fact, GBM is considered radioresistant $(3)$, and many failures after radiotherapy (RT) occur 'in-field' (4). This means that novel strategies to increase the efficacy of RT, such as radiosensitization, are necessary.

Epigenetic aberrations, such as DNA methylation and histone protein modifications, are critical for carcinogenesis and cancer growth $(5,6)$. Most cancer cells harbor hypermethylated $\mathrm{CpG}$ islands in their DNA, and DNA methyltransferases (DNMTs) mediate the introduction of a methyl-group $(\mathrm{CH} 3)$ at cytosine bases $(7,8)$. Furthermore, critical biological pathways in gliomas, such as the p53 or retinoblastoma pathways, are known to be affected by the hypermethylation of $\mathrm{CpG}$ islands $(9,10)$, while methylation of the $\mathrm{O}^{6}$-methylguanine-DNA methyltransferase (MGMT) promoter has a significant impact on predicting the outcome of GBM patients following chemoradiation $(2,11)$.

Given the frequent overexpression of DNMT in cancer, DNMT inhibitors (DNMTis) have been widely investigated as potential targets of anti-cancer therapy, with a radiosensitizing effect in various cancer cell lines (12-16). We have previously reported psammaplin A, a nonnucleoside DNMTi, as a radiosensitizer in human GBM cells (12). However, further application of the compound in vivo was hindered due to its poor biostability (17). For this reason, we have been investigating the application of nonnucleoside DNMTis with respect to their radiosensitizing 
effect as well as their biostability. Herein we present the results of a novel DNMTi synthesized using a phthalimidoalkanamide backbone.

\section{Materials and Methods}

MA17 and cell culture. A phthalimido-alkanamide derivative (undisclosed formula, $\mathrm{C}_{26} \mathrm{H}_{30} \mathrm{~N}_{2} \mathrm{O}_{5}$ ), was synthesized at the College of Pharmacy, in the Catholic University of Daegu, using a phthalimid backbone (PubChem Compound ID: 6809). The compound was designated as 'MA17'. When 6-week old albino male ICR mice $(\mathrm{n}=4)$ were injected with $10 \mathrm{mg} / \mathrm{kg}$ of MA17, this showed good bioavailability with an elimination half-life of $1.0 \pm 0.2$ hour (IACUC No. 2016-007, unpublished data communicated from the Catholic University of Daegu) (Table I). MA17 was dissolved in DMSO at a concentration of $20 \mathrm{mM}$ and was stored at room temperature.

Four human GBM cell lines (U87MG, U373MG, U138MG, and T98G) and one normal human astrocyte (NHA) cell line were used in this study. U87MG/U373MG cells (Korean Cell Line Bank, Seoul, Korea) and U138MG/T98G/NHA (American Type Culture Collection, Manassas, USA) cells were cultured and maintained in RPMI 1640 and DMEM media, respectively (Welgene, Daegu, Korea), at $37^{\circ} \mathrm{C}$ with $5 \%$ (v/v) $\mathrm{CO}_{2}$. Media were supplemented with $10 \%$ fetal bovine serum (Gibco, Grand Island, US) with an added $12.5 \mu \mathrm{g} / \mathrm{ml}$ of gentamicin (Gibco). U87MG and U373 cells represent GBM cells with methylation in the MGMT promoter, whereas $\mathrm{U} 138 \mathrm{MG}$ and $\mathrm{T} 98 \mathrm{G}$ cells have an unmethylated $M G M T$ promoter in the current study (18-20).

Clonogenic assay. Cell survival after RT \pm MA17 was measured using the clonogenic assay as previously reported (12). Cells were trypsinized from the growing monolayer cultures, and the planned number of cells were seeded into T25 flasks and were incubated for $24 \mathrm{~h}$ prior RT. For RT, 6 megavoltage (MV) photon beams were used at doses of 0-8 Gy (Clinac 21iX Silhouette, Varian Medical Systems, Palo Alto, USA). For RT+MA17, MA17 was administered for 24 hours prior to RT. Following treatment, cells were incubated for 14 days to allow for colony formation. Colonies containing $\geq 50$ cells were counted after fixation with methanol and staining with $0.5 \%$ crystal violet. All experiments were done in triplicates.

Tumor growth delay assay. Tumor growth delay assays were performed in vivo using the U87MG and U138MG cell lines, under the approval of the Seoul National University Hospital (IACUC No. 16-0016-C1A1). Tumor cells $\left(2 \times 10^{6}\right)$ in $100 \mu$ l of media supplemented with fetal bovine serum were injected intradermally in the back of 6-week old female BALB/C-nude mice (Orient, Seongnam-si, Korea) irrespective of their weight. The mean weight of mice was $18.19 \pm 0.92 \mathrm{~g}$. U87MG and U138MG cells were transferred in RPMI and DMEM media, respectively, and were supplemented with $15 \%$ fetal bovine serum. When the mean tumor volume reached $450 \mathrm{~mm}^{3}$ for $\mathrm{U} 87 \mathrm{MG}$ and $300 \mathrm{~mm}^{3}$ for $\mathrm{U} 138 \mathrm{MG}$, mice were randomly placed into four groups: i) control (arm 1,

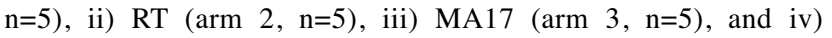
RT+MA17 (arm 4, n=5).

After randomizing the mice in the aforementioned groups, RT was delivered to mice in arms 2 and 4 . In each mouse, one fraction of 8-Gy was delivered to the tumor at a dose rate of 300 monitor units/min using 6-MV electron beams. The back of the mouse was
Table I. Pharmacokinetic parameters after intravenous injection of compound MA17 at a dose of $10 \mathrm{mg} / \mathrm{kg}(\mathrm{n=4})$.

\begin{tabular}{lc}
\hline Parameters & Mean $\pm \mathrm{SD}$ \\
\hline $\mathrm{t}_{1 / 2}, \lambda \mathrm{n}($ hour $)$ & $1.0 \pm 0.2$ \\
$\mathrm{C}_{0}(\mathrm{ng} / \mathrm{mL})$ & $6458.2 \pm 1818.2$ \\
$\mathrm{AUC}_{\text {all }}(\mathrm{ng} \cdot \mathrm{hr} / \mathrm{mL})$ & $941.3 \pm 206.7$ \\
$\mathrm{AUC}$ & $947.5 \pm 205.2$ \\
$\mathrm{CL}(\mathrm{mL} / \mathrm{min} / \mathrm{hg})$ & $184.0 \pm 49.9$ \\
$\mathrm{~V}_{\mathrm{SS}}(\mathrm{L} / \mathrm{kg})$ & $3.3 \pm 2.1$ \\
\hline
\end{tabular}

$\mathrm{SD}$ : Standard deviation; $\mathrm{t}_{1 / 2}$ : the average terminal elimination half; $\mathrm{C}_{0}$ : the concentration at the beginning of observation; CL: the systemic clearance; AUC: the area under the curve; $\mathrm{V}_{\mathrm{SS}}$ : the volume of distribution at steady state.

covered with 1-cm tissue-equivalent bolus to ensure the coverage of the superficial portion of the tumors. Vehicle or MA17 was delivered twice intraperitoneally pre-RT and 45 minutes post-RT. The vehicle consisted of $477.78 \mu \mathrm{L}$ phosphate-buffered saline and $22.22 \mu \mathrm{L}$ DMSO for each injection, whereas the drug for arms 3 and 4 consisted of 3-mg MA17 dissolved in the identical solution. Therefore, mice in arms 3 and 4 received a total MA17 of $6 \mathrm{mg}$ each. Starting from the first day of the treatment, the tumor volume was measured every 2-3 days, using a digital caliper (Digimatic Caliper CD-15CPX, Mitutoyo Corporation, Kawasaki, Japan) and was calculated using the formula of $\{$ (length $\times$ width $\times$ height $) \times \pi\} / 6$. Tumor doubling time (TBT) was used to compare the tumor growth between treatment arms.

DNMT1 colorimetric assay. A quantitative assay for the DNMT1 inhibitory activity of MA17 was performed using the colorimetric EpiQuik DNMT activity/inhibition assay Ultra kit (catalog No. P3009, Epigentek, Farmingdale, USA) and $100 \mathrm{ng}$ of DNMT1 proteins (catalog No. E-15000, Epigentek), according to the manufacturer's instructions. The DNMT1 activity was proportional to the sample optical density (OD). The DNMT1 inhibition was calculated as follows: DNMT1 inhibition $(\%)=[1-$ (inhibitor sample OD - blank OD)/(no inhibitor sample OD - blank OD)] $\times 100$. Data were obtained from triplicate experiments.

Western blotting. The expression of each target protein was compared to that of $\beta$-actin by densitometric analysis, using the ChemiDoc Touch Image Lab (Bio-Medical Science Co., Seoul, Korea). To evaluate the inhibitory effect of MA17 on DNMT1, DNMT3A, and DNMT3B, comparisons of these proteins' expressions were performed between cells treated with and without MA17 for 24 hours. The expression of cleaved caspase-3 (antibody from Cell Signaling Technology, Danvers, MA, US) was examined to evaluate apoptosis. Western blotting for DNMT1, DNMT3A, DNMT3B, and cleaved caspase-3 expression was performed as previously reported (12). To investigate how MA17 affects autophagy, we selected 2 target proteins, LC3B and Beclin-1, which are known to accurately represent autophagosome formation (21). LC3-I to LC3-II conversion is an indicator of autophagic activity in mammals, represented by the LC3-II to LC3-I ratio (21). Primary antibodies against LC3B (1:1000; catalog No. 3868; Cell Signaling Technology) and Beclin-1 (1:1000; catalog No. 3495; Cell Signaling Technology) were used. Cleaved caspase-3, LC3-II to LC3-I ratio, 


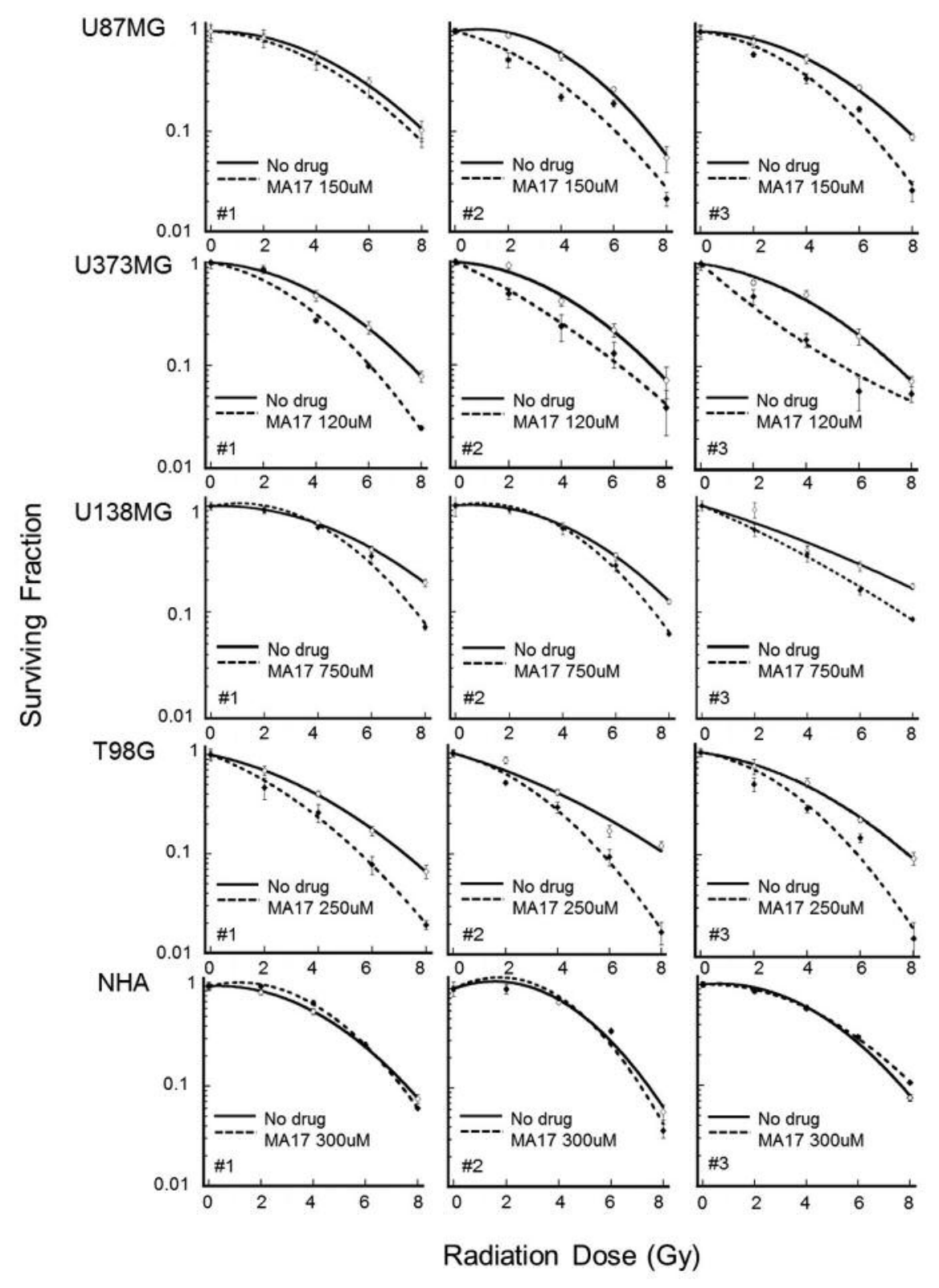

Figure 1. Clonogenic cell survival in four GBM cell lines and in NHA cells following radiotherapy combined with MA17 or on its own. Cells were treated with MA17 for 24 h before radiotherapy with graded doses of X-ray. Surviving fractions are shown as mean \pm standard deviation from triplicate experiments.

and Beclin-1 expression was examined and compared between cells treated with MA17 for $24 \mathrm{~h}$ prior to RT at these times post-RT: i) 0.5 , ii) 6 , iii) 24 , and iv) $48 \mathrm{~h}$ and untreated cells. RT consisted of $6 \mathrm{~Gy}$ in a single fraction using $6 \mathrm{MV}$ photons.

In a separate experiment from our lab using human lung adenocarcinoma cells (A549, Korean Cell Line Bank) and next generation sequencing, cells treated with RT+MA17 showed a significant down-regulation in the Fanconi anemia pathway, specifically in the regulation of the FANCA gene, compared to those treated by RT alone. Fanconi anemia pathway is known as one of the key pathways for the repair of DNA double-strand breaks (22). Therefore, to validate the additional inhibition of FANCA gene and the Fanconi anemia pathway by MA17, we evaluated the expression of FANCA proteins in the four GBM cells in a timely manner as above by western blotting ( $\mathrm{n}=1$ for each cell).

Immunocytochemistry. Immunocytochemistry was performed as described previously using the DAPI nuclear counterstain and a FITC-labeled secondary antibody (Invitrogen, Camarillo, US) (12). Evaluated timepoints were post-RT 0.5, 6, 24, and $48 \mathrm{~h}$. Slides were 


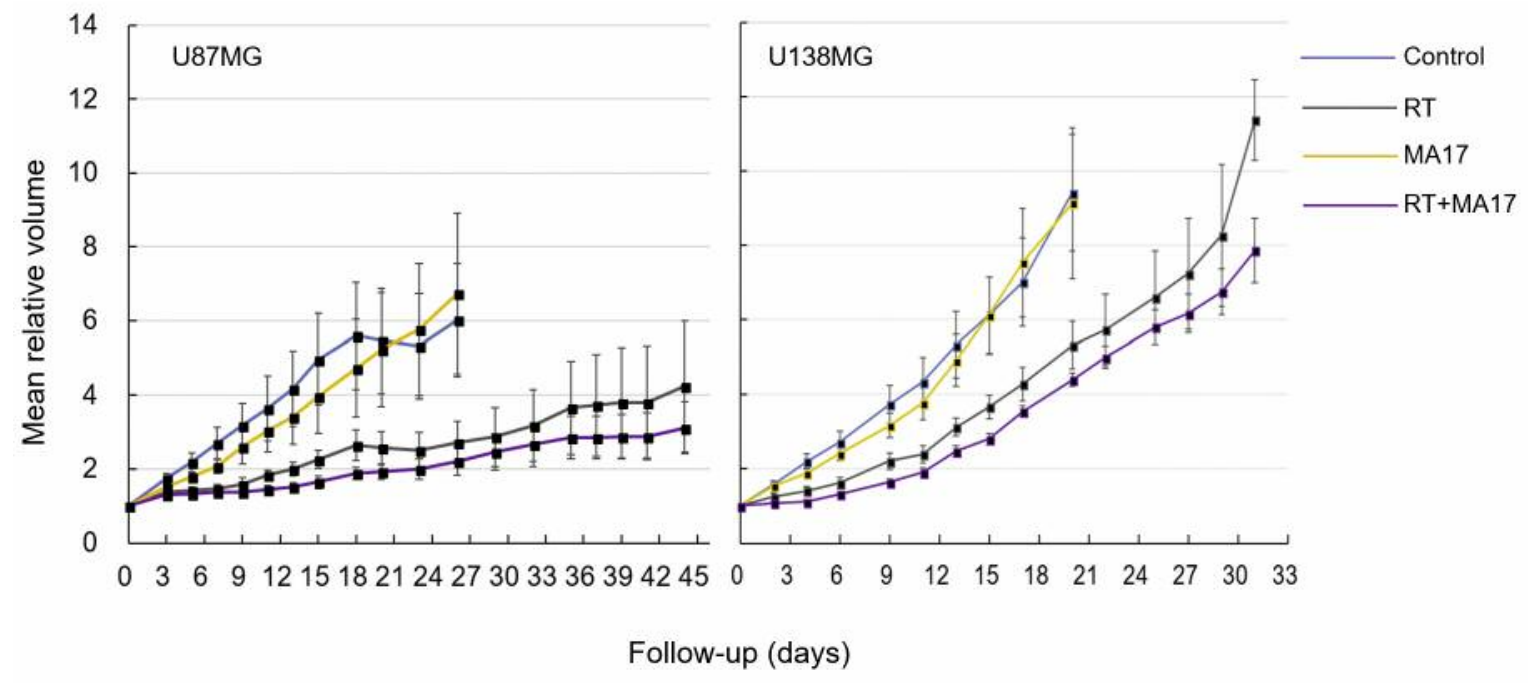

Figure 2. Tumor growth curves of U87MG and U138MG after treatment in BALB/C-nude mice. Tumor volumes are presented as relative volumes when the volume on the day of treatment is normalized to 1. Error bar: Standard error.

examined using a confocal laser scanning microscope (LSM 800, Carl Zeiss, Oberkochen, Germany). Images were acquired using the acquisition software ZEN 2.3 (blue edition) (Carl Zeiss). Proportion of cells harboring $>10 \gamma \mathrm{H} 2 \mathrm{AX}$ foci were counted to examine DNA double-strand breaks over time. A single fraction of 6-Gy was delivered for all cells.

Statistical analysis. The graphic and data analysis application KaleidaGraph, version 3.51 (Synergy Software, Reading, USA), was used to fit the surviving fractions to a linear quadratic model. From this model, parameters were obtained to calculate the required dose to achieve a surviving fraction of 0.2 . Then, to evaluate the radiosenstitizing effect of MA17 in vivo, a one-tailed ratio paired t-test was used between the required radiation doses to achieve the specified surviving fraction. Sensitizer enhancement ratio (SER) was obtained using the following formula: $S E R=(R T-$ dose required for surviving fraction 0.2 without MA17) $\div$ (RTdose required for surviving fraction 0.2 with MA17). In the tumor growth delay assay, the one-tailed Mann-Whitney test was used to compare the mean TBTs between arms 1, 2, 3, and 4. All statistical analyses were performed using the Statistical Package for Social Sciences, version 23.0 (IBM Corp., Armonk, USA). It was considered statistically significant when the $p$-value was below 0.05. All results are presented as a mean \pm standard deviation, unless specified differently.

\section{Results}

Radiosensitization in vitro. The 50\% inhibitory concentrations of MA17, which is the concentration of MA17 needed to kill half of the cells, were $171.81 \pm 22.82$, 108.29 $\pm 29.96, \quad 774.86 \pm 57.59, \quad 246.17 \pm 75.06, \quad$ and $324.66 \pm 44.55 \mu \mathrm{M}$ in U87MG, U373MG, U138MG, T98G, and NHA cells, respectively. Therefore, in the assays that followed, we established a MA17 concentration of 150, 120,
750, 250, and $300 \mu \mathrm{M}$ for using in U87MG, U373MG, U138MG, T98G, and NHA cells, respectively.

MA17 was shown to significantly radiosensitize all four human GBM cell lines with the 50\% inhibitory doses mentioned above, however, it did not affect the radiation sensitivity of NHA cells (Figure 1). The mean SERs of MA17 at a surviving fraction of 0.2 in U87MG, U373MG, U138MG, T98G, and NHA cells were $1.196(p=0.034)$, $1.441(p=0.029), 1.152(p=0.030), 1.350 \quad(p<0.001)$, and $1.016(p=0.420)$, respectively.

Radiosensitization in vivo. Tumor growth curves starting on the day of randomization and treatment are displayed in Figure 2. The mice harboring U138MG cells were observed for a shorter time period and were sacrificed earlier, since the tumor grew faster than the U87MG cells.

In mice with U87MG cells the mean TDT measured was $6.51 \pm 5.73,12.25 \pm 2.96,11.50 \pm 9.19$, and $18.60 \pm 4.84$ days for arms 1, 2, 3, and 4, respectively (arm $1 v s$. arm 2, $p=0.071 ; \operatorname{arm} 1 v s$. arm 3, $p=0.088 ; \operatorname{arm} 1 v s$. $\operatorname{arm} 4$, $p=0.007 ; \operatorname{arm} 2 v s$. arm 3, $p=0.312 ; \operatorname{arm} 2$ vs. $\operatorname{arm} 4$, $p=0.009$; arm $3 v s$. arm 4, $p=0.054$ ) (Figure 3A). The mean TDT was significantly longer in arm 4 compared to arm 2 with a margin of 6.35 days.

In mice harboring U138MG cells the mean TDT was $3.67 \pm 1.09,8.26 \pm 2.31,4.63 \pm 1.19$, and $11.32 \pm 1.56$ days in arms 1, 2, 3, and 4, respectively ( $\operatorname{arm} 1 \mathrm{vs.}$ arm 2, $p=0.007$; arm 1 vs. arm 3, $p=0.126$; arm 1 vs. arm 4, $p=0.002$; arm 2 vs. arm 3, $p=0.014 ;$ arm 2 vs. arm 4, $p=0.030$; arm 3 vs. arm $4, p=0.002$ ) (Figure 3B). The mean TDT was significantly prolonged in arm 4 with the addition of MA17 by 3.06 days, as compared to arm 2 . 
(A) U87MG

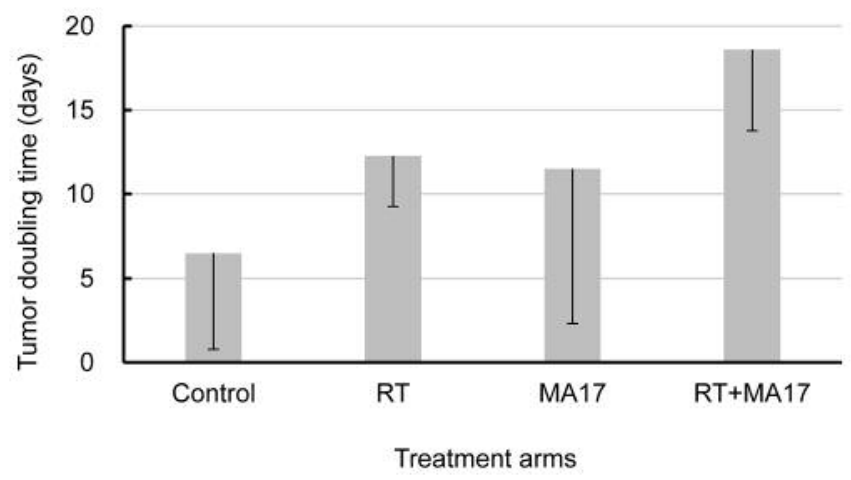

(B) U138MG

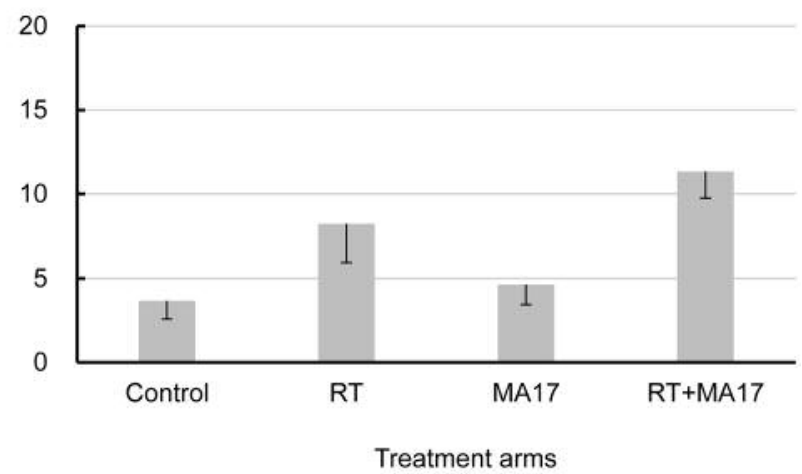

Figure 3. Mean tumor doubling times of (A) U87MG and (B) U138MG in vivo by treatment arms. RT, radiotherapy. Error bar: standard deviation.

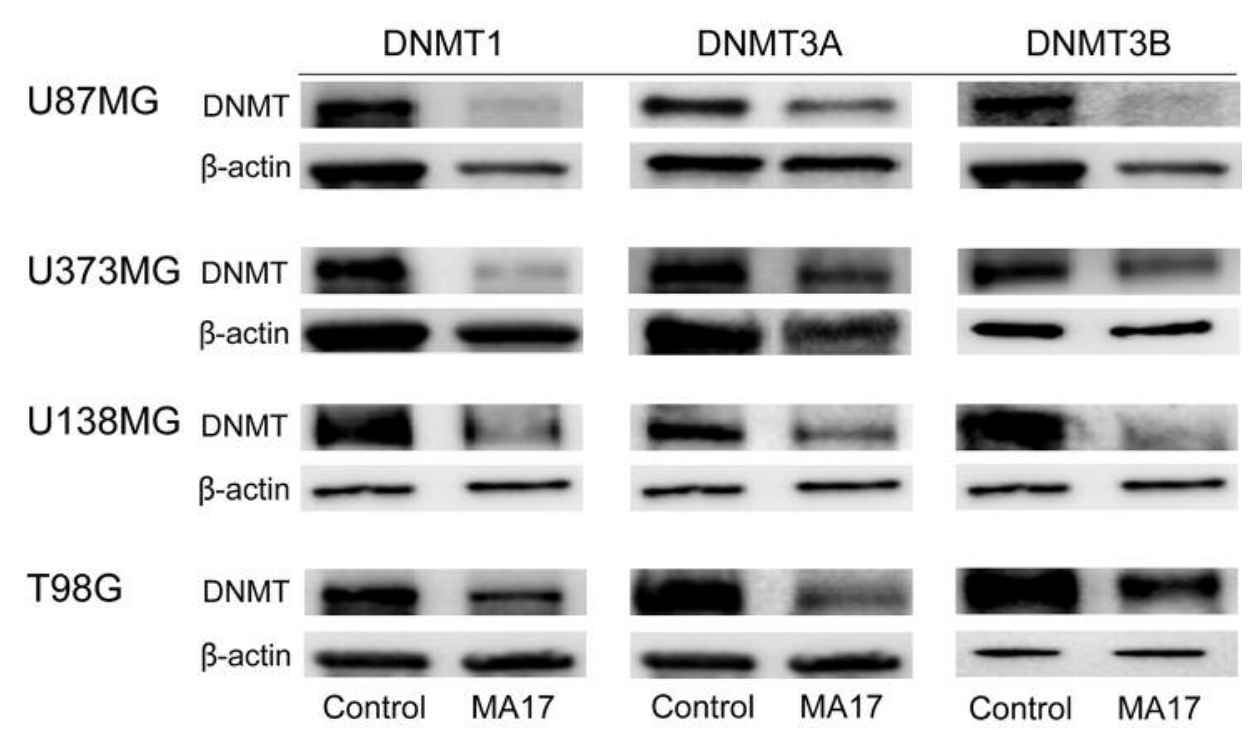

Figure 4. Western blot results of DNMT 1, 3A, and 3B in 4 glioblastoma cell lines after treatment with or without MA17. DNMT, DNA methyltransferase.

DNMT inhibition. According to the colorimetric assay, DNMT1 activity was inhibited by $60.29 \pm 28.22 \%$ when MA17 was used. Western blotting confirmed that the expression of DNMT1, DNMT3A, and DNMT3B proteins was inhibited in all four GBM cell lines by MA17, as compared to vehicle control treatment (Figure 4).

Apoptosis and autophagy. Evaluation of cleaved caspase-3 expression by western blotting revealed that early apoptotic tumor cell death between $0.5-24 \mathrm{~h}$ post-RT was significantly higher in U87MG and U373MG cells treated with RT+MA17, as compared to those receiving RT alone (Figure 5). In contrast, in U138MG and T98G cells, although cleaved caspase- 3 expression levels were still higher when cells were treated with RT+MA17, compared to those not receiving MA17, the difference was only noticeable during a later phase of $24-48 \mathrm{~h}$ post-RT (Figure 5).

The densitometric analyses of LC3-II/LC3-I ratio and Beclin-1 expression are displayed in Figure 6. In U87MG and U373MG cells the addition of MA17 increased the LC3II/LC3-I ratio and the Beclin-1 expression at an early phase of 0.5-6 hours post-RT (Figure 6). In U138MG cells and T98G cells the LC3-I to LC3-II ratio did not differ between cells treated by RT compared to RT+MA17, and was not consistent throughout the triplicate repeats, whereas the expression of Beclin-1 protein was higher in cells receiving MA17. 


\section{U87MG}

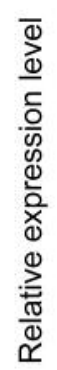

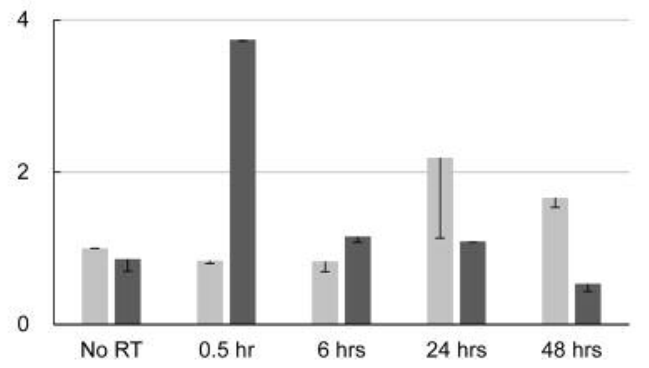

U373MG

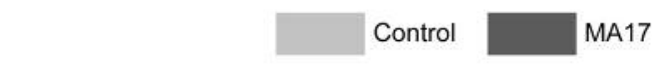

\section{Post-RT time}

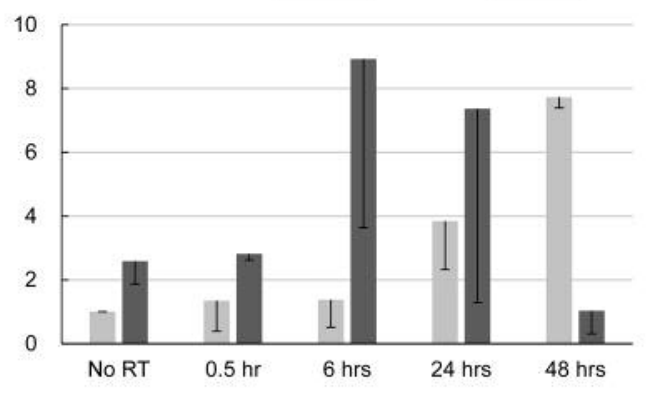

\section{U138MG}

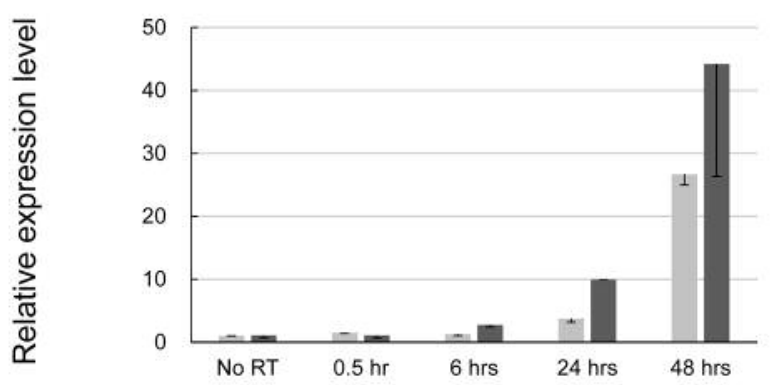

T98G

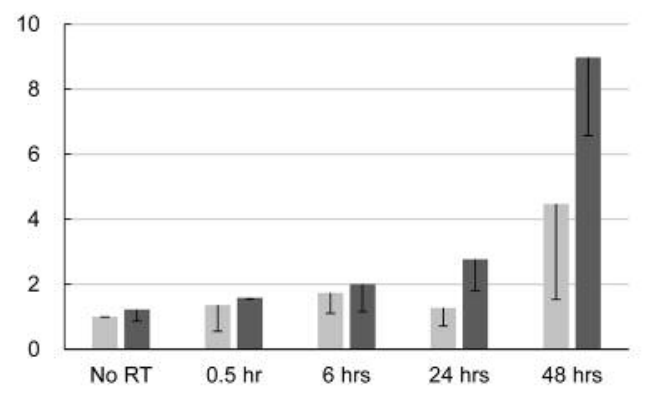

Post-RT time

Figure 5. Densitometric analysis of western blot results for cleaved caspase-3 expression normalized to $\beta$-actin in four glioblastoma cell lines treated with RT $\pm M A 17$. RT, Radiotherapy. Light gray bar, RT; Dark gray bar, RT+MA17. Error bar: standard deviation.

Double-stranded DNA breaks. Changes in the proportion of cells harboring $>10 \gamma \mathrm{H} 2 \mathrm{AX}$ foci are shown in Figure 7 and Figure 8 . The proportion of cells with double-stranded DNA breaks was similar between the treatment arms for the first $6 \mathrm{~h}$ post-RT, independent of MA17 treatment. However, during a later phase of 24-48 h post-RT, a notably larger proportion of cells harbored $>10 \gamma \mathrm{H} 2 \mathrm{AX}$ foci if they had been treated with MA17, compared to those exposed to the RT treatment alone.

FANCA inhibition. In all four GBM cell lines, the expression of FANCA proteins was lower when they were treated with RT+MA17, compared to cells treated with RT alone (Figure 9). The difference was notable especially at 0.5 and 6 hours postRT for all cell lines but it progressively decreased thereafter. Interestingly, in U87MG and T98G cells, a significant inhibition of FANCA was sustained up to $48 \mathrm{~h}$ post-RT.

\section{Discussion}

GBM demonstrates a poor median survival of patients below 2 years $(1,2)$. Therefore, strategies that can enhance the therapeutic window of RT by sensitizing tumor cells to the RT tumoricidal effects are extremely valuable.

Several DNMTis have been tested as potential radiosensitizing agents during the past decade in various cancer cells, such as from the brain, lung, colorectum, stomach, head and neck, pancreas, and prostate (12-16) and have demonstrated some promising results in vitro. Possible mechanisms of radiosensitization by DNMTis include the following: i) inhibition of DNA repair process following RT, ii) decrease of the repopulation rate of cancer cells during RT by preferentially killing proliferative cells, and iii) induction of apoptosis in cancer cells (26). However, despite the optimistic in vitro results, their clinical use as radiosensitizers have been hampered by either their toxicity or poor biostability $(17,27)$. For example, in our previous study, a non-nucleoside DNMTi, psammaplin A, showed significant radiosensitizing effects in GBM and lung cancer cells (12). However, the half-life of the agent in the mouse serum was present for under 10 minutes, limiting its clinical use in that form (17). This means that there is a huge need for novel DNMTis, which can sensitize cancer cells to radiation without affecting normal tissues, and possess 


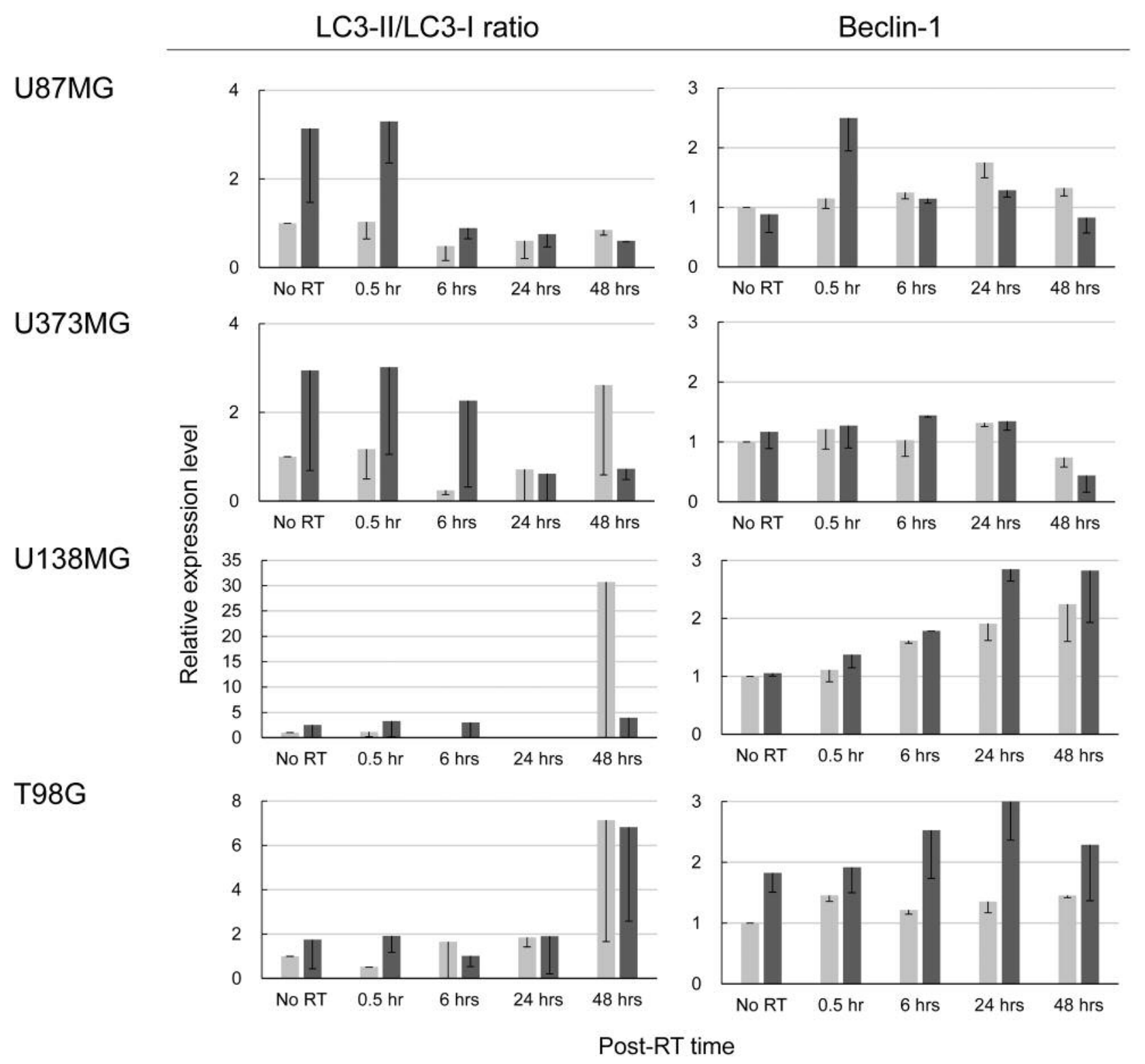

Figure 6. Densitometric analysis of the LC3-II to LC3-I ratio and of Beclin-1 expression normalized to $\beta$-actin in four glioblastoma cell lines treated with RT \pm MA17. RT, Radiotherapy. Light gray bar, RT; Dark gray bar, RT+MA17. Error bar: standard deviation.

stability of a certain duration in vivo, in order to maximize the therapeutic effect of RT in its clinical application.

In our study, we evaluated a novel phthalimido-alkanamide derivative, as a DNMTi as well as a radiosensitizer, using four different GBM cell lines. Western blot results revealed that MA17 is an inhibitor of DNMT1, DNMT3A, and DNMT3B. Pretreatment with MA17 significantly enhanced the radiosensitivity of the U87MG, U373MG, U138MG, and T98G cells in vitro. In contrast, MA17 did not show any evidence of radiosensization in the astrocytic NHA cells, demonstrating an ideal behavior as a radiosensitizer. A subsequent tumor growth delay assay, using the U87MG and U138MG cells, also revealed that injections of MA17 pre- or post-RT may generate a significant delay in GBM cell growth in vivo, compared to those treated with RT alone. Of note, it is very promising that MA17 has demonstrated significant radiosensitization even in GBM cells with unmethylated MGMT (U138MG), given their radioresistance and persistent survival $(2,11)$.

The significant radiosensitization of GBM cells when treated with MA17 may be attributed to different reasons. Firstly, the rate of apoptosis increased in all four cell lines treated with $\mathrm{RT}+\mathrm{MA17}$, as compared to cells treated with RT alone, 

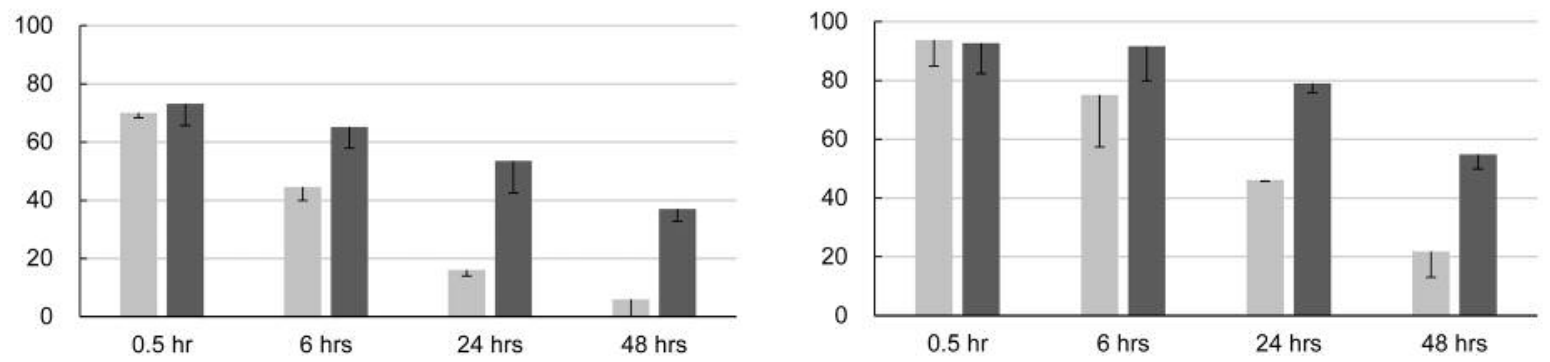

Post-RT time

\section{U138MG}
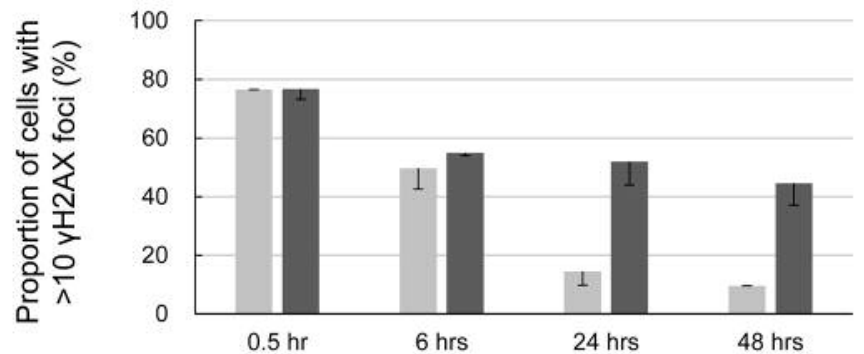

T98G

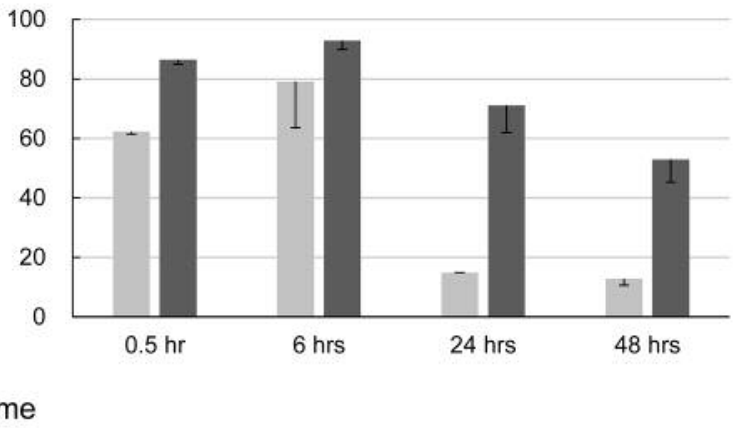

Figure 7. Immunocytochemistry results to evaluate changes in the proportion of cells harboring $>10 \gamma H 2 A X$ foci in four glioblastoma cell lines treated with RT \pm MA17. RT, Radiotherapy. Light gray bar, RT; Dark gray bar, RT+MA17. Error bar: standard deviation.

although the timing and the magnitude of enhanced apoptosis differed between the different GBM cell lines we used. Apoptotic cell death is one of the major cell-killing mechanisms of RT (28), and it is well known that some DNMTis affect RT-induced apoptosis. For instance, Qiu et al. have reported that 5-aza-CdR, a DNMTi, enhanced RT-induced apoptosis in gastric cancer cells causing a cell cycle arrest at the $\mathrm{G}_{2}-\mathrm{M}$ phase (14). Kim et al. have also demonstrated that psammaplin A enhances RT-induced apoptosis in human lung cancer cells (12). Secondly, our results revealed that MA17 also enhances RT-induced autophagy in GBM cells. Autophagy is a critical process involved in cell death (29) and is known to be induced by RT in many types of cancers (30). Several studies have shown that combining drugs that enhance autophagy with RT, or even regulating genes associated with autophagy, can enhance the radiosensitivity of cancer cells (30). Moreover, it is known that epigenetic alterations may affect the choice of cells for autophagic cell death (31). Although LC3-I to LC3-II conversion was dominantly seen in cells with MGMT methylation (U87MG and U373MG), whereas Beclin-1 function was dominantly elevated in cells without MGMT methylation, the explanation for this difference is yet to be defined. Nevertheless, RT-induced autophagy was enhanced to some degree with the use of MA17 in all cell lines. Thirdly, double-stranded DNA break foci were observed for a prolonged period in cells treated with MA17 in addition to RT. The difference in proportion of cells possessing increased numbers of $\gamma \mathrm{H} 2 \mathrm{AX}$ foci was especially noted 24-48 hours post-RT in all four GBM cell lines. In another study from our group, the use of A549 cells and the KEGG pathway, as well as the Fanconi anemia pathway, which plays a critical role in repairing double-stranded DNA breaks (32), was significantly down-regulated with the use of MA17 (unpublished data). For this reason, we tested whether the expression of FANCA protein is down-regulated in GBM cells when these were pretreated with MA17. Indeed, the expression of FANCA was significantly lower in GBM cells receiving MA17, which probably contributed to the suppression of double-stranded DNA breaks. Although not validated at a transcriptome level by RNA sequencing, MA17 seems to suppress the Fanconi anemia pathway in GBM cells, like with lung cancer cells, resulting in their radiosensitization. 


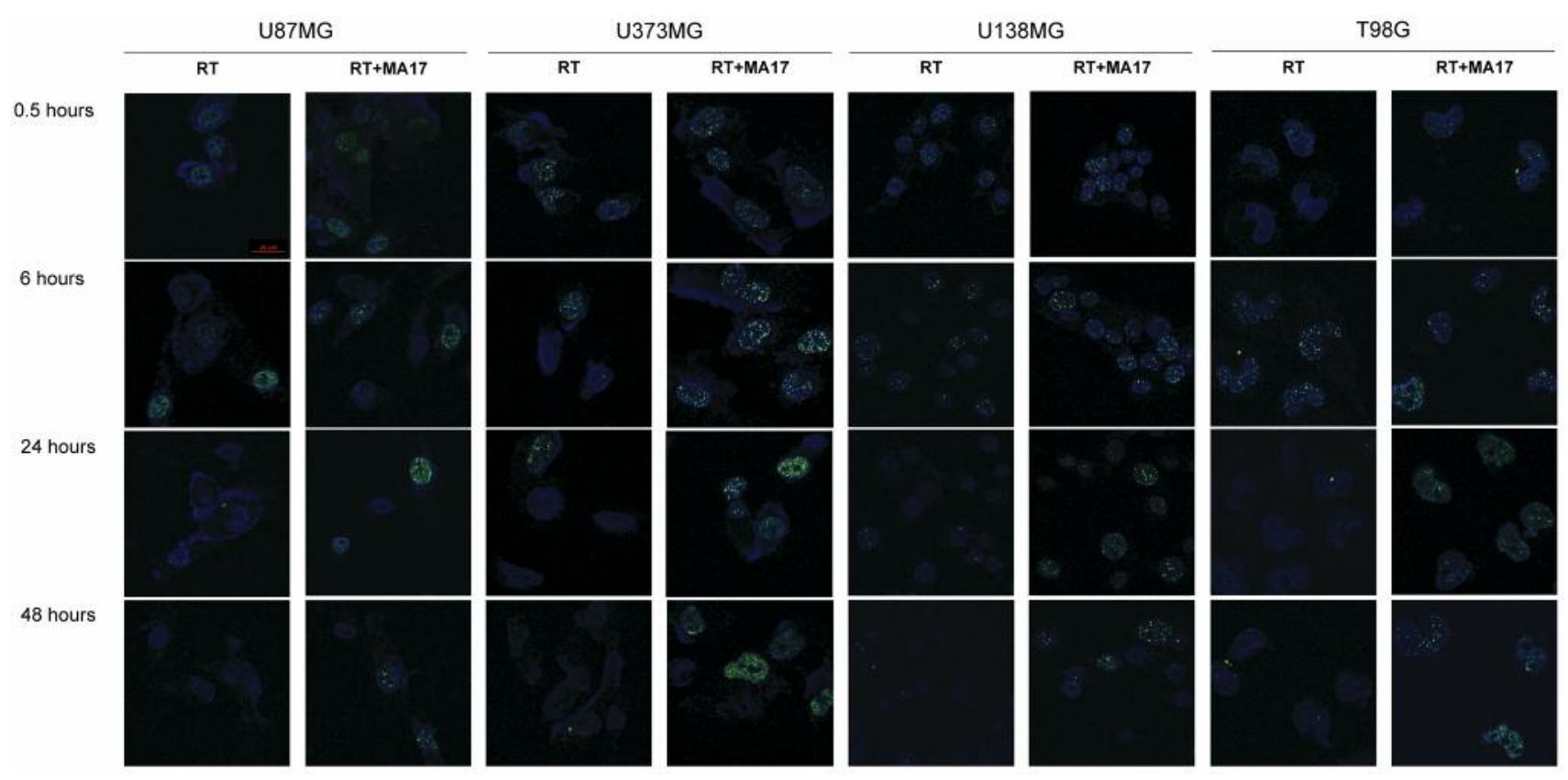

Figure 8. Radiation-induced $\gamma H 2 A X$ foci. Representative micrographs were obtained from 4 glioblastoma cell lines treated by RT \pm MA17. Cells harboring $>10 \gamma H 2 A X$ foci are significantly well visualized at post-RT 24-48 h in cells treated with MA17. Blue fluorescence, DAPI; Green fluorescence, $\gamma H 2 A X$.

U87MG

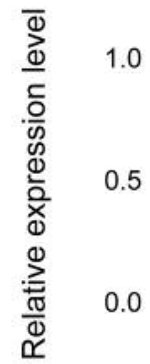$$
0.0
$$<smiles>CCC</smiles>

\section{U373MG}

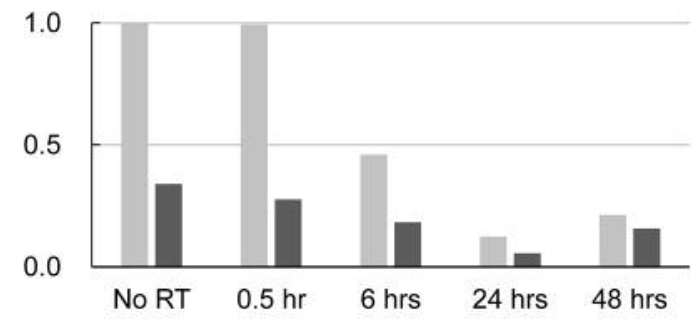

Post-RT time

\section{U138MG}

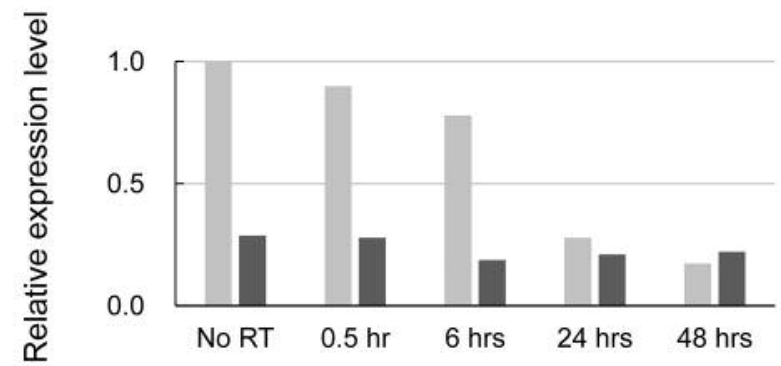

T98G

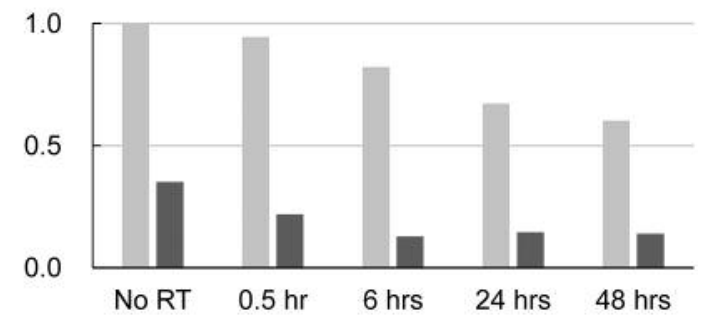

Post-RT time

Figure 9. Densitometric analysis of FANCA expression normalized to $\beta$-actin in four glioblastoma cell lines treated with $R T \pm M A 17$ ( $n=1)$. RT, Radiotherapy. Light gray bar, RT; Dark gray bar, RT+MA17. 
Several obstacles need to be surmounted for this novel phthalimido-alkanamide derivative to be tested in clinical trials for GBM patients. The agent is highly lipophilic and has shown low solubility in saline. As a consequence, MA17 was injected in mice after being dissolved in DMSO, an agent associated with neurotoxicity (33). Furthermore, although injected intraperitoneally, the exact concentration of the drug in the serum and brain tissues of the mice was not examined in our study. Therefore, the proper form of the drug administration as well as its concentration still needs to be determined. The toxicity profiles of the drug also need to be evaluated. Although MA17 did not affect the sensitivity to RT in NHA cells, and while non-nucleoside DNMTis are less toxic compared to nucleosides in general, unexpected toxicity of the drug may be encountered. In our hands, no acute toxicity seemed to occur in mice after administration of MA17 during the follow-up period.

In summary, we used a newly-synthesized agent that exhibits significant radiosensitizing effects in GBM cells without affecting normal astrocytes. Apoptosis, autophagy, and suppression of DNA repair is enhanced by this agent in combination with RT. Several limitations for clinical application of the drug still exist. Nevertheless, this novel compound brightens the prospects of treating GBM, which is currently regarded as an incurable type of cancer.

\section{Conflicts of Interest}

The Authors declare no conflicts of interest.

\section{Authors' Contributions}

CWW, JHK, HJK, BSS, EM, and IHK participated in study design. CWW and SYS participated in cell culture, clonogenic assay, and DNMT inhibitions assay. ESM participated in compound synthesis. BSS participated in the pharmacokinetic analysis. Data analysis was done by CWW, JHK, HCK, HJK, and IHK. CWW initially drafted the manuscript. EM and IHK edited the manuscript. All authors read and approved the final manuscript.

\section{Acknowledgements}

This study was supported by the Korean government (MSIP) through the National Research Foundation of Korea grant 2013M2A2A7043683 (Il Han Kim), and the Seoul National University Hospital through SNUH CRI 0320170080 (Il Han Kim) and SNUH CRI 0320130400 (Hak Jae Kim).

\section{References}

1 Stupp R, Mason WP, van den Bent MJ, Weller M, Fisher B, Taphoorn MJ, Belanger K, Brandes AA, Marosi C, Bogdahn U, Curschmann J, Janzer RC, Ludwin SK, Gorlia T, Allgeier A, Lacombe D, Cairncross JG, Eisenhauer E and Mirimanoff RO: Radiotherapy plus concomitant and adjuvant temozolomide for glioblastoma. N Engl J Med 352(10): 987-996, 2005.
2 Wee CW, Kim E, Kim N, Kim IA, Kim TM, Kim YJ, Park CK, Kim JW, Kim CY, Choi SH, Kim JH, Park SH, Choe G, Lee ST, Chang JH, Kim SH, Suh CO and Kim IH: Novel recursive partitioning analysis classification for newly diagnosed glioblastoma: A multi-institutional study highlighting the MGMT promoter methylation and IDH1 gene mutation status. Radiother Oncol 123(1): 106-111, 2017.

3 Bao S, Wu Q, McLendon RE, Hao Y, Shi Q, Hjelmeland AB, Dewhirst MW, Bigner DD and Rich JN: Glioma stem cells promote radioresistance by preferential activation of the DNA damage response. Nature 444(7120): 756-760, 2006.

4 Minniti G, Amelio D, Amichetti M, Salvati M, Muni R, Bozzao A, Lanzetta G, Scarpino S, Arcella A and Enrici RM: Patterns of failure and comparison of different target volume delineations in patients with glioblastoma treated with conformal radiotherapy plus concomitant and adjuvant temozolomide. Radiother Oncol 97(3): 377-381, 2010.

5 Jones PA and Baylin SB: The epigenomics of cancer. Cell 128(4): 683-692, 2007.

6 Rodriguez-Paredes $\mathrm{M}$ and Esteller M: Cancer epigenetics reaches mainstream oncology. Nat Med 17(3): 330-339, 2011.

7 van Engeland M, Derks S, Smits KM, Meijer GA and Herman JG: Colorectal cancer epigenetics: complex simplicity. J Clin Oncol 29(10): 1382-1391, 2011.

8 Feinberg AP and Tycko B: The history of cancer epigenetics. Nat Rev Cancer 4(2): 143-153, 2004.

9 Amatya VJ, Naumann U, Weller M and Ohgaki H: TP53 promoter methylation in human gliomas. Acta Neuropathol 110(2): 178-184, 2005.

10 Nakamura M, Yonekawa Y, Kleihues P and Ohgaki H: Promoter hypermethylation of the RB1 gene in glioblastomas. Lab Invest 81(1): 77-82, 2001.

11 Hegi ME, Diserens AC, Gorlia T, Hamou MF, de Tribolet N, Weller M, Kros JM, Hainfellner JA, Mason W, Mariani L, Bromberg JE, Hau P, Mirimanoff RO, Cairncross JG, Janzer RC and Stupp R: MGMT gene silencing and benefit from temozolomide in glioblastoma. N Engl J Med 352(10): 997$1003,2005$.

$12 \mathrm{Kim}$ HJ, Kim JH, Chie EK, Young PD, Kim IA and Kim IH: DNMT (DNA methyltransferase) inhibitors radiosensitize human cancer cells by suppressing DNA repair activity. Radiat Oncol 7: 39, 2012.

13 Hofstetter B, Niemierko A, Forrer C, Benhattar J, Albertini V, Pruschy M, Bosman FT, Catapano CV and Ciernik IF: Impact of genomic methylation on radiation sensitivity of colorectal carcinoma. Int J Radiat Oncol Biol Phys 76(5): 1512-1519, 2010.

14 Qiu H, Yashiro M, Shinto O, Matsuzaki T and Hirakawa K: DNA methyltransferase inhibitor 5-aza-CdR enhances the radiosensitivity of gastric cancer cells. Cancer Sci 100(1): 181188, 2009.

15 De Schutter H, Kimpe M, Isebaert S and Nuyts S: A systematic assessment of radiation dose enhancement by 5-Aza-20deoxycytidine and histone deacetylase inhibitors in head-andneck squamous cell carcinoma. Int J Radiat Oncol Biol Phys 73(3): 904-912, 2009.

16 Dote H, Cerna D, Burgan WE, Carter DJ, Cerra MA, Hollingshead MG, Camphausen K and Tofilon PJ: Enhancement of in vitro and in vivo tumor cell radiosensitivity by the DNA methylation inhibitor zebularine. Clin Cancer Res 11(12): 45714579, 2005. 
17 Kim HJ, Kim TH, Seo WS, Yoo SD, Kim IH, Joo SH, Shin S, Park ES, Ma ES and Shin BS: Pharmacokinetics and tissue distribution of psammaplin A, a novel anticancer agent, in mice. Arch Pharm Res 35(10): 1849-1854, 2012.

18 Alonso MM, Gomez-Manzano C, Bekele BN, Yung WK and Fueyo $\mathrm{J}$ : Adenovirus-based strategies overcome temozolomide resistance by silencing the $\mathrm{O}^{6}$-methylguanine-DNA methyltransferase promoter. Cancer Res 67(24): 11499-11504, 2007.

19 Gaspar N, Marshall L, Perryman L, Bax DA, Little SE, VianaPereira M, Sharp SY, Vassal G, Pearson AD, Reis RM, Hargrave D, Workman P and Jones C: MGMT-independent temozolomide resistance in pediatric glioblastoma cells associated with a PI3kinase-mediated HOX/stem cell gene signature. Cancer Res 70(22): 9243-9252, 2010.

20 Li S, Chou AP, Chen W, Chen R, Deng Y, Phillips HS, Selfridge J, Zurayk M, Lou JJ, Everson RG, Wu KC, Faull KF, Cloughesy T, Liau LM and Lai A: Overexpression of isocitrate dehydrogenase mutant proteins renders glioma cells more sensitive to radiation. Neuro Oncol 15(1): 57-68, 2013.

21 Klionsky DJ, Cuervo AM and Seglen PO: Methods for monitoring autophagy from yeast to human. Autophagy 3(3): 181-206, 2007.

22 Walden $\mathrm{H}$ and Deans AJ: The Fanconi anemia DNA repair pathway: structural and functional insights into a complex disorder. Annu Rev Biophys 43: 257-278, 2014.

23 Gilbert MR, Wang M, Aldape KD, Stupp R, Hegi ME, Jaeckle KA, Armstrong TS, Wefel JS, Won M, Blumenthal DT, Mahajan A, Schultz CJ, Erridge S, Baumert B, Hopkins KI, Tzuk-Shina T, Brown PD, Chakravarti A, Curran WJ Jr. and Mehta MP: Dose-dense temozolomide for newly diagnosed glioblastoma: a randomized phase III clinical trial. J Clin Oncol 31(32): 40854091, 2013.

24 Gilbert MR, Dignam JJ, Armstrong TS, Wefel JS, Blumenthal DT, Vogelbaum MA, Colman H, Chakravarti A, Pugh S, Won M, Jeraj R, Brown PD, Jaeckle KA, Schiff D, Stieber VW, Brachman DG, Werner-Wasik M, Tremont-Lukats IW, Sulman EP, Aldape KD, Curran WJ Jr. and Mehta MP: A randomized trial of bevacizumab for newly diagnosed glioblastoma. N Engl J Med 370(8): 699-708, 2014
25 Kong DS, Nam DH, Kang SH, Lee JW, Chang JH, Kim JH, Lim YJ, Koh YC, Chung YG, Kim JM and Kim CH: Phase III randomized trial of autologous cytokine-induced killer cell immunotherapy for newly diagnosed glioblastoma in Korea. Oncotarget 8(4): 7003-7013, 2017.

26 Smits KM, Melotte V, Niessen HE, Dubois L, Oberije C, Troost EG, Starmans MH, Boutros PC, Vooijs M, van Engeland M and Lambin P: Epigenetics in radiotherapy: where are we heading? Radiother Oncol 111(2): 168-177, 2014.

27 Aparicio A and Weber JS: Review of the clinical experience with 5-azacytidine and 5-aza-2'-deoxycytidine in solid tumors. Curr Opin Investig Drugs 3(4): 627-633, 2002.

28 Dewey WC, Ling CC and Meyn RE: Radiation-induced apoptosis: relevance to radiotherapy. Int J Radiat Oncol Biol Phys 33(4): 781-796, 1995.

29 Ito H, Daido S, Kanzawa T, Kondo S and Kondo Y: Radiationinduced autophagy is associated with LC3 and its inhibition sensitizes malignant glioma cells. Int J Oncol 26(5): 1401-1410, 2005.

30 XinY, Jiang F, Yang C, Yan Q, Guo W, Huang Q, Zhang L and Jiang G: Role of autophagy in regulating the radiosensitivity of tumor cells. J Cancer Res Clin Oncol 143(11): 2147-2157, 2017.

31 Sui X, Zhu J, Zhou J, Wang X, Li D, Han W, Fang Y and Pan $\mathrm{H}$ : Epigenetic modifications as regulatory elements of autophagy in cancer. Cancer Lett 360(2): 106-113, 2015.

32 Michl J, Zimmer J and Tarsounas M: Interplay between Fanconi anemia and homologous recombination pathways in genome integrity. EMBO J 35(9): 909-923, 2016.

33 Yuan C, Gao J, Guo J, Bai L, Marshall C, Cai Z, Wang L and Xiao M: Dimethyl sulfoxide damages mitochondrial integrity and membrane potential in cultured astrocytes. PLoS One 9(9): e107447, 2014
Received December 17, 2018

Revised January 6, 2019

Accepted January 22, 2019 